\title{
Synthesis and structural characterization of $\mathrm{Na}_{2} \mathrm{MnP}_{2} \mathrm{O}_{7}$ crystal
}

\author{
N K LOKANATH, M A SRIDHAR, J SHASHIDHARA PRASAD*, G S GOPALAKRISHNA ${ }^{\dagger}$ \\ and K G ASHAMANJARI \\ Department of Studies in Physics, ${ }^{\dagger}$ Department of Studies in Geology, University of Mysore, Manasagangotri, \\ Mysore 570 006, India
}

MS received 15 November 1999

\begin{abstract}
Na}_{2} \mathrm{MnP}_{2} \mathrm{O}_{7}$ crystals were synthesized by hydrothermal technique. Crystals obtained are in the form of single crystals of rhombohedral morphology with lattice parameters as follows: triclinic, $P \overline{1}, \lambda=0 \cdot 71069 \AA$, $a=6.657(3) \AA, b=6.714(6) \AA, c=6.518(4) \AA, \alpha=112.31(6)^{\circ}, \beta=92.14(4)^{\circ}, \gamma=83.89(5)^{\circ}, V=268.0(3) \AA^{3}, Z=2$, $\rho_{\text {cal }}=2,3 \cdot 121 \mathrm{~g} / \mathrm{cm}^{3}, \mu=3 \cdot 121 \mathrm{~mm}^{-1}, F_{000}=244$, goodness-of-fit on $F=1 \cdot 348$, final $R$ indices with $[I>3 \sigma(I)]$ $R=0.051$ and $\omega R=0.065$.
\end{abstract}

Keywords. NASICON; hydrothermal; crystal structure.

\section{Introduction}

NASICON compounds were the most popular materials available for many years as source materials for high temperature battery devices. But the interest in these compounds has slowly declined because of their polycrystallinity, non-stoichiometric composition, complex conduction mechanism and host of other problems (Byrappa and Gopalakrishna 1986). Owing to these problems, scientists looked into new compounds known as NASICON analogues, which are pure phosphates and are almost free from the NASICON problems (Yoshimura et al 1981; Byrappa et al 1985a, b, 1986; Clearfield et al 1986; Lokanath et al 1999). However, these compounds always have only the triorthophosphate end members and their structures are directly related to $\mathrm{Na}_{3} \mathrm{Sc}_{2} \mathrm{P}_{3} \mathrm{O}_{12}$ (Byrappa and Gopalakrishna 1986; Lokanath et al 1999). We have reported for the first time high ionic conductivity in condensed phosphates which are much easier to synthesize in the form of single crystals with stoichiometric composition (Byrappa et al 1989; Salvador Gali et al 1989, 1992). We report here the synthesis and crystal structure of a new material which promises to be a good solid electrolyte.

\section{Experimental}

Crystals of the compound $\mathrm{Na}_{2} \mathrm{MnP}_{2} \mathrm{O}_{7}$ were synthesized in teflon liners by the hydrothermal method using Morey

\footnotetext{
*Author for correspondence
}

type autoclave at $220-250^{\circ} \mathrm{C}$ and $50-60$ bars. The starting mixture consists of manganese oxide, $85 \%$ orthophosphoric acid and $2 \cdot 5-5 \mathrm{M} \mathrm{NaOH}$ solution which acts as a mineralizer.

$\mathrm{Na}_{2} \mathrm{MnP}_{2} \mathrm{O}_{7}$ crystals could be synthesized under the following molar ratio

$$
\mathrm{Na}_{2} \mathrm{O}: \mathrm{MnO}: \mathrm{P}_{2} \mathrm{O}_{5}=3-5: 0 \cdot 5-0 \cdot 75: 12-16 \text {. }
$$

The following reaction explains the synthesis of the compound

$$
\begin{aligned}
\mathrm{MnCl}_{2}+2 \mathrm{H}_{3} \mathrm{PO}_{4}+2 \mathrm{NaOH} \rightarrow & \mathrm{Na}_{2} \mathrm{MnP}_{2} \mathrm{O}_{7} \\
& +2 \mathrm{HCl}+3 \mathrm{H}_{2} \mathrm{O}
\end{aligned}
$$

Light purple coloured crystals with sub vitreous lustre were obtained in the form of single crystals having sizes ranging from $1-3 \mathrm{~mm}$, with well developed morphology.

Rhombohedral shaped crystal with approximate size $0.1 \times 0.1 \times 0.15 \mathrm{~mm}$ was mounted on a Rigaku AFC7S diffractometer equipped with a graphite monochromated MoK $\alpha$ X-ray source $(\lambda=0.71069 \AA)$. The unit cell parameters were obtained by using the method of short vectors followed by least squares refinement of 19 reflections. Lorentz and polarization corrections were applied.

The crystal belongs to the triclinic space group $P \overline{1}$. The structure was solved using SIR92 (Altomare et al 1993). The structure was refined using teXsan (Molecular Structure Corporation, USA 1995) for 923 unique reflections. The refinement was carried out by keeping all oxygen atoms as isotropic and the rest as anisotropic. The 
procedure is similar to the structure solutions of compounds reported earlier (Byrappa et al 1985a, b; Lokanath et al 1999). 101 parameters were refined using 923 observed reflections with $I>3 \sigma(I)$ to $R=0.051$ and $\omega R=0 \cdot 065$.

\section{Results and discussion}

The positional parameters and equivalent temperature factors are given in table 1 . Anisotropic parameters $\left(U_{\mathrm{ij}}\right)$ are listed in table 2. Table 3 gives the bond distances and

Table 1. Atomic coordinates and equivalent thermal parameters of the nonhydrogen atoms.

\begin{tabular}{lrrrr}
\hline Atom & $x$ & $y$ & $z$ & $U_{\text {eq }}$ \\
\hline Mnl & $0 \cdot 6503(2)$ & $0 \cdot 1145(2)$ & $0 \cdot 2253(2)$ & $0 \cdot 0111(3)$ \\
P1 & $1 \cdot 1469(3)$ & $0 \cdot 2212(3)$ & $0 \cdot 2531(3)$ & $0 \cdot 0096(5)$ \\
P2 & $1 \cdot 6269(3)$ & $0 \cdot 6335(3)$ & $0 \cdot 3242(3)$ & $0 \cdot 0097(5)$ \\
Na1 & $1 \cdot 1507(6)$ & $0 \cdot 7443(6)$ & $0 \cdot 2135(8)$ & $0 \cdot 046(1)$ \\
O1 & $1.4306(8)$ & $0 \cdot 7431(8)$ & $0 \cdot 4348(8)$ & $0 \cdot 014(1)$ \\
O2 & $1 \cdot 7204(8)$ & $0 \cdot 7773(8)$ & $0 \cdot 2227(9)$ & $0 \cdot 015(1)$ \\
O3 & $1 \cdot 7843(7)$ & $0 \cdot 6173(8)$ & $0 \cdot 5127(8)$ & $0 \cdot 012(1)$ \\
O4 & $1 \cdot 0920(7)$ & $0 \cdot 3693(8)$ & $0 \cdot 1226(8)$ & $0 \cdot 013(1)$ \\
O5 & $1 \cdot 3267(7)$ & $0 \cdot 0587(8)$ & $0 \cdot 1441(8)$ & $0 \cdot 012(1)$ \\
O6 & $0 \cdot 9716(7)$ & $0 \cdot 1136(8)$ & $0 \cdot 2873(9)$ & $0 \cdot 016(1)$ \\
O7 & $0 \cdot 6249(7)$ & $0 \cdot 4052(8)$ & $0 \cdot 1605(8)$ & $0 \cdot 013(1)$ \\
\hline
\end{tabular}

Table 2. Anisotropic thermal parameters of the non-hydrogen atoms.

\begin{tabular}{|c|c|c|c|c|c|c|}
\hline Atom & $U_{11}$ & $U_{22}$ & $U_{33}$ & $U_{12}$ & $U_{13}$ & $U_{23}$ \\
\hline Mnl & $0 \cdot 0115(7)$ & $0 \cdot 0085(7)$ & $0 \cdot 0095(7)$ & $-0.0000(4)$ & $-0 \cdot 0005(4)$ & $-0.0006(5)$ \\
\hline P1 & $0 \cdot 0104(10)$ & $0 \cdot 0065(10)$ & $0 \cdot 008(1)$ & 0.0001(7) & $-0.0012(7)$ & $-0.0013(8)$ \\
\hline P2 & $0 \cdot 0130(10)$ & $0 \cdot 0059(10)$ & $0 \cdot 008(1)$ & $-0 \cdot 0006(7)$ & $-0 \cdot 0015(7)$ & $-0.0003(8)$ \\
\hline Nal & $0 \cdot 043(2)$ & $0 \cdot 018(2)$ & $0 \cdot 076(3)$ & $-0.008(2)$ & $-0 \cdot 039(2)$ & $0 \cdot 017(2)$ \\
\hline $\mathrm{O} 1$ & $0 \cdot 019(3)$ & $0 \cdot 014(3)$ & $0 \cdot 006(3)$ & $0 \cdot 000(2)$ & $-0 \cdot 002(2)$ & $0 \cdot 000(2)$ \\
\hline $\mathrm{O} 2$ & $0 \cdot 022(3)$ & $0 \cdot 005(3)$ & $0 \cdot 016(3)$ & $0 \cdot 001(2)$ & $0 \cdot 000(2)$ & $0 \cdot 001(2)$ \\
\hline $\mathrm{O} 3$ & $0 \cdot 014(3)$ & $0 \cdot 009(3)$ & $0 \cdot 007(3)$ & $0 \cdot 001(2)$ & $-0 \cdot 004(2)$ & $-0 \cdot 003(2)$ \\
\hline $\mathrm{O} 4$ & $0 \cdot 013(3)$ & $0 \cdot 010(3)$ & $0 \cdot 014(3)$ & $0 \cdot 002(2)$ & $-0 \cdot 000(2)$ & $0 \cdot 002(2)$ \\
\hline O5 & $0 \cdot 014(3)$ & $0 \cdot 010(3)$ & $0 \cdot 007(3)$ & $0 \cdot 001(2)$ & $-0 \cdot 001(2)$ & $-0 \cdot 001(2)$ \\
\hline O6 & $0 \cdot 013(3)$ & $0 \cdot 011(3)$ & $0 \cdot 021(3)$ & $-0 \cdot 001(2)$ & $-0 \cdot 007(2)$ & $0 \cdot 004(2)$ \\
\hline $\mathrm{O} 7$ & $0 \cdot 021(3)$ & $0 \cdot 004(3)$ & $0 \cdot 010(3)$ & $-0.003(2)$ & $-0.005(2)$ & $-0.001(2)$ \\
\hline
\end{tabular}

Table 3. Bond lengths (Å).

\begin{tabular}{lrlr}
\hline Atoms & Length & Atoms & Length \\
\hline Mnl-O1 & $2 \cdot 118(5)$ & P2-O1 & $1 \cdot 494(5)$ \\
Mnl-O2 & $2 \cdot 256(5)$ & P2-O2 & $1 \cdot 549(5)$ \\
Mnl-O5 & $2 \cdot 235(5)$ & P2-O3 & $1 \cdot 612(5)$ \\
Mnl-O5 & $2 \cdot 244(5)$ & P2-O7 & $1 \cdot 500(5)$ \\
Mnl-O6 & $2 \cdot 162(5)$ & Nal-O1 & $2 \cdot 315(6)$ \\
Mnl-O7 & $2 \cdot 137(5)$ & Nal-O4 & $2 \cdot 429(6)$ \\
P1-Nal & $3 \cdot 111(4)$ & Nal-O4 & $2 \cdot 592(6)$ \\
P1-O3 & $1 \cdot 585(5)$ & Nal-O5 & $2 \cdot 707(6)$ \\
P1-O4 & $1 \cdot 545(5)$ & Nal-O6 & $2 \cdot 511(6)$ \\
P1-O5 & $1 \cdot 525(5)$ & Nal-O7 & $2 \cdot 698(7)$ \\
P1-O6 & $1 \cdot 502(5)$ & & \\
\hline
\end{tabular}




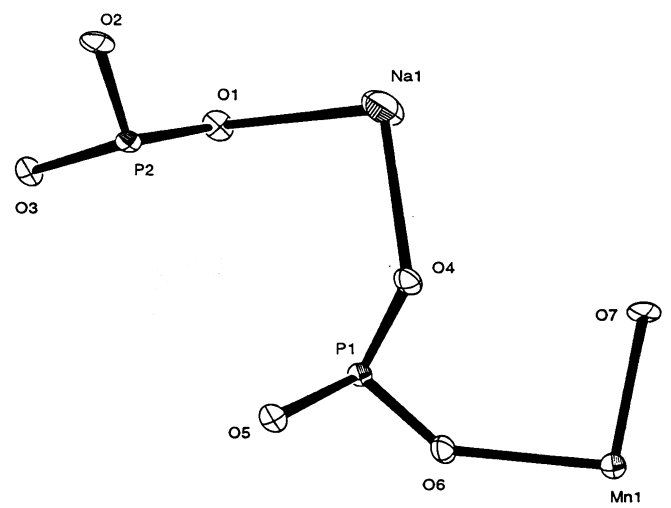

Figure 1. ORTEP of the molecule at 50\% probability.

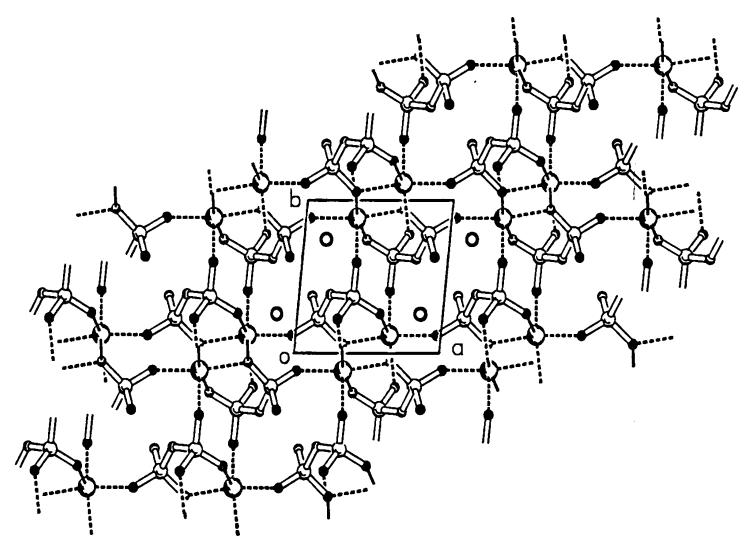

Figure 2. Packing of molecules down $c$ axis.

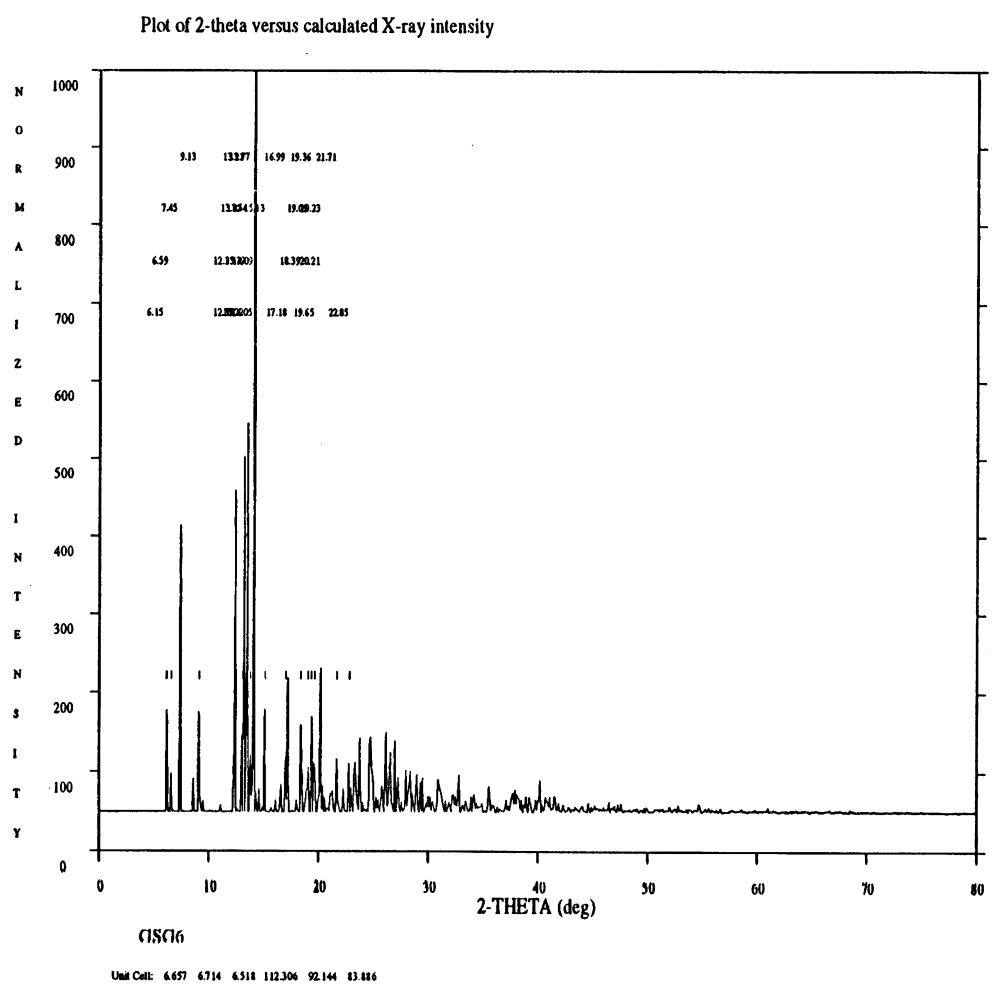

Figure 3. Powder pattern of the sample. 
Table 4. Bond angles $\left({ }^{\circ}\right)$.

\begin{tabular}{|c|c|c|c|}
\hline Atoms & Angle & Atoms & Angle \\
\hline O1-Mnl-O2 & $94 \cdot 9(2)$ & P1-Nal-O7 & $92 \cdot 2(2)$ \\
\hline O1-Mnl-O5 & $88 \cdot 7(2)$ & O1-Nal-O4 & $96 \cdot 4(2)$ \\
\hline O1-Mnl-O5 & $168 \cdot 4(2)$ & O1-Nal-O4 & $160 \cdot 7(2)$ \\
\hline O1-Mnl-O6 & $94 \cdot 4(2)$ & O1-Nal-O5 & $83 \cdot 2(2)$ \\
\hline O1-Mnl-O7 & $97 \cdot 5(2)$ & O1-Nal-O6 & $114 \cdot 2(2)$ \\
\hline O2-Mnl-O5 & $91 \cdot 1(2)$ & O1-Nal-O7 & $92 \cdot 7(2)$ \\
\hline O2-Mnl-O5 & $83 \cdot 0(2)$ & O4-Nal-O4 & $71 \cdot 7(2)$ \\
\hline O2-Mnl-O6 & $80 \cdot 2(2)$ & O4-Nal-O5 & $152 \cdot 7(3)$ \\
\hline O2-Mnl-O7 & $166 \cdot 9(2)$ & O4-Nal-O6 & $142 \cdot 6(2)$ \\
\hline O5-Mnl-O5 & $80 \cdot 0(2)$ & O4-Nal-O7 & $85 \cdot 9(2)$ \\
\hline O5-Mnl-O6 & $171 \cdot 0(2)$ & O4-Nal-O5 & $100 \cdot 5(2)$ \\
\hline O5-Mnl-O7 & $93 \cdot 5(2)$ & O4-Nal-O6 & $83 \cdot 1(2)$ \\
\hline O5-Mnl-O6 & $96 \cdot 4(2)$ & O4-Nal-O7 & $71 \cdot 9(2)$ \\
\hline O5-Mnl-O7 & $85 \cdot 7(2)$ & O5-Nal-O6 & $57 \cdot 4(2)$ \\
\hline O6-Mnl-O7 & $94 \cdot 5(2)$ & O5-Nal-O7 & $66 \cdot 9(2)$ \\
\hline Nal-P1-O3 & $114 \cdot 5(2)$ & O6-Nal-O7 & $112 \cdot 4(2)$ \\
\hline Nal-P1-O4 & $142 \cdot 0(2)$ & Mnl-O1-P2 & $130 \cdot 9(3)$ \\
\hline Nal-P1-O5 & $60 \cdot 5(2)$ & Mnl-O1-Nal & $110 \cdot 7(2)$ \\
\hline Nal-P1-O6 & $53 \cdot 0(2)$ & P2-O1-Nal & $118 \cdot 2(3)$ \\
\hline O3-P1-O4 & $103 \cdot 5(3)$ & $\mathrm{Mnl}-\mathrm{O} 2-\mathrm{P} 2$ & $136 \cdot 5(3)$ \\
\hline $\mathrm{O} 3-\mathrm{P} 1-\mathrm{O} 5$ & $108 \cdot 5(3)$ & $\mathrm{P} 1-\mathrm{O} 3-\mathrm{P} 2$ & $135 \cdot 7(3)$ \\
\hline O3-P1-O6 & $108 \cdot 6(3)$ & P1-O4-Nal & $129 \cdot 9(3)$ \\
\hline O4-P1-O5 & $110 \cdot 0(3)$ & P1-O4-Nal & $120 \cdot 8(3)$ \\
\hline O4-P1-O6 & $113 \cdot 6(3)$ & $\mathrm{Nal}-\mathrm{O} 4-\mathrm{Nal}$ & $108 \cdot 3(2)$ \\
\hline O5-P1-O6 & $112 \cdot 2(3)$ & Mnl-O5-Mnl & $100 \cdot 0(2)$ \\
\hline $\mathrm{O} 1-\mathrm{P} 2-\mathrm{O} 2$ & $110 \cdot 1(3)$ & Mnl-O5-Pl & $124 \cdot 8(3)$ \\
\hline $\mathrm{O} 1-\mathrm{P} 2-\mathrm{O} 3$ & $108 \cdot 1(3)$ & Mnl-O5-Nal & $122 \cdot 6(2)$ \\
\hline O1-P2-O7 & $116 \cdot 5(3)$ & Mnl-O5-P1 & $121 \cdot 7(3)$ \\
\hline $\mathrm{O} 2-\mathrm{P} 2-\mathrm{O} 3$ & $105 \cdot 0(3)$ & Mnl-O5-Nal & $95 \cdot 1(2)$ \\
\hline O2-P2-O7 & $111 \cdot 6(3)$ & $\mathrm{P} 1-\mathrm{O} 5-\mathrm{Nal}$ & $90 \cdot 2(2)$ \\
\hline O3-P2-O7 & $104 \cdot 8(3)$ & Mnl-O6-P1 & $139 \cdot 1(3)$ \\
\hline P1-Nal-O1 & $96 \cdot 6(2)$ & Mnl-O6-Nal & $113 \cdot 9(2)$ \\
\hline P1-Nal-O4 & $166 \cdot 9(2)$ & P1-O6-Nal & $98 \cdot 5(3)$ \\
\hline P1-Nal-O4 & $95 \cdot 4(2)$ & Mnl-O7-P2 & $128 \cdot 1(3)$ \\
\hline P1-Nal-O5 & $29 \cdot 3(1)$ & $\mathrm{Mnl}-\mathrm{O} 7-\mathrm{Nal}$ & $98 \cdot 0(2)$ \\
\hline P1-Nal-O6 & $28 \cdot 5(1)$ & P2-O7-Nal & $121 \cdot 4(3)$ \\
\hline
\end{tabular}

angles. Figure 1 represents ORTEP (Molecular Structure Corporation, USA 1995) diagram of the molecule with thermal ellipsoids at $50 \%$ probability. The packing of molecules down $c$ axis is shown in figure 2 . The material is a good example of a three-dimensional conductor. Figure 3 represents the simulated powder pattern of the sample.

\section{Acknowledgements}

The authors would like to express their thanks to DST, New Delhi, for financial assistance under the projects SP/I2/FOO/93 and SP/S2/M-44/96.

\section{References}

Altomare A, Cascarano M, Giacovazzo C and Guagliardi A 1993 J. Appl. Cryst. 26343

Byrappa K and Gopalakrishna G S 1986 Prog. Cryst. Growth \& Charact. 1189

Byrappa K, Kulkarni A B, Gopalakrishna G S, Venkatachalapathy V and Viswanathaiah M N 1985a J. Mater. Sci. Lett. 4565

Byrappa K, Kulkarni A B, Gopalakrishna G S and Venkatachalapathy V 1985b J. Less Common Metals 110359

Byrappa K, Gopalakrishna G S and Kulkarni A B 1986 J. Cryst. Growth 79232

Byrappa K, Gopalakrishna G S and Salvador Gali 1989 Indian J. Phys. A63 321

Clearfield A, Subramanian M A, Rudolf P R and Moin A 1986 Solid State Ionics 18/19 13

Lokanath N K, Sridhar M A, Shashidhara Prasad J, Gopalakrishna G S and Ashamanjari K G 1999 J. Mater. Sci. Lett. 181723

Molecular Structure Corporation, teXan, Single Crystal Structure Analysis Software 1995 Version 1.7, MSC, 3200 Research Forest Drive, The Woodlands, TX 77381, USA

Salvador Gali, Byrappa K and Gopalakrishna G S 1989 Acta Crystallogr. C45 1667

Salvador Gali, Carddenas A, Byrappa K and Gopalakrishna G S 1992 Acta Crystallogr. C48 1650

Yoshimura M, Fujii K and Somiya S 1981 Mater. Res. Bull. 16 327 